\title{
Prediction of moisture content uniformity using hyperspectral imaging technology during the drying of maize kernel**
}

\author{
Min Huang ${ }^{1,2}$, Weiyan Zhao ${ }^{1}$, Qingguo Wang, Min Zhang ${ }^{2 *}$, and Qibing Zhu \\ ${ }^{1}$ Key Laboratory of Advanced Process Control for Light Industry (Ministry of Education), \\ ${ }^{2}$ State Key Laboratory of Food Science and Technology; Jiangnan University, Wuxi, Jiangsu, China, 214122
}

Received August 31, 2014; accepted January 8, 2015

\begin{abstract}
A b s t r a c t. Moisture content uniformity is one of critical parameters to evaluate the quality of dried products and the drying technique. The potential of the hyperspectral imaging technique for evaluating the moisture content uniformity of maize kernels during the drying process was investigated. Predicting models were established using the partial least squares regression method. Two methods, using the prediction value of moisture content to calculate the uniformity (indirect) and predicting the moisture content uniformity directly, were investigated. Better prediction results were achieved using the direct method (with correlation coefficients $R_{P}=0.848$ and root-mean-square error of prediction $\mathrm{RMSEP}=2.73)$ than the indirect method $\left(\mathrm{R}_{\mathrm{p}}=0.521\right.$ and RMSEP $=10.96)$. The hyperspectral imaging technique showed significant potential in evaluating moisture content uniformity of maize kernels during the drying process.

$\mathrm{K}$ e y w o r d s: maize kernels, hyperspectral imaging, moisture content uniformity, drying
\end{abstract}

\section{INTRODUCTION}

Drying is a mass transfer process consisting of removal of water or another solvent by evaporation from the material. This process is important and often used as a final production step before selling or packaging products. In the past decades, many drying techniques have been widely reported and used in food manufacturing. For a drying process, drying uniformity is one of important parameters to evaluate the quality of dried products and drying technology. Drying uniformity is defined as the relative standard deviation (RSD, ratio of standard deviation to mean measurement value) of temperature, moisture content (MC), colour, and shrinkage

*Corresponding author e-mail: min@jiangnan.edu.cn

**This work was financial supported from China 863 HI-TECH R and D Program (No. 2011AA100802), National Natural Science Foundation of China (Grant No. 61271384 and 1275155), the 111 Project (B12018) and PAPD of Jiangsu Higher Education Institutions and sponsored by Qing Lan Project, 2014-2016.
(Wang et al., 2013a; 2013b). Among them, moisture content uniformity (MCU) was gradually concerned by scholars. MC non-uniformity can cause many disadvantages, such as relatively short safe storage life, reduce storage stability, and can even cause mildew deterioration and reduce product use value at serious non-uniformity (Hashemi and Murray Douglas, 2003). Generally, MCU is calculated by the definition (ratio of MC standard deviation to MC mean measurementvalue). Thenumberofselected samples determines the accuracy of uniformity measurement. The more selected samples, the higher uniformity. Since the traditional laboratory methods for MC measurements of agricultural foods, the gravimetric oven method and Karl Fisher titration (Aguilera, 2003), are destructive measurements, the same samples cannot be used for further analysis, which limits the number of selected samples for calculating uniformity.

Rapid non-destructive technologies for measuring the drying qualities of agricultural products have been studied extensively (Faustino et al., 2007; Fernández et al., 2005; Nowak and Lewicki, 2005; Mendoza et al., 2006; Toyoda et al., 2001). Among these approaches, machine vision and near-infrared spectroscopy are the two main methods (Lucas et al 2008; Makky et al., 2014; Mireei et al., 2010; Romano et al., 2012; Wu et al., 2010). However, the machine vision method can only acquire average image information within the visible range and nearinfrared spectroscopy can only acquire spectral information and cannot obtain the spatial information of the samples. As a relatively novel non-destructive technology, hyperspectral imaging integrates the advantages of machine vision and near-infrared spectroscopy, while overcoming the

(C) 2015 Institute of Agrophysics, Polish Academy of Sciences 
drawbacks of both techniques when used alone. Hyperspectral imaging provides more detailed or complete information, including internal structure characteristics, morphological information, and chemical composition (Huang et $a l ., 2013)$. This technology has been applied to non-destructive measurement of agricultural products for evaluating internal quality (Ariana and $\mathrm{Lu}, 2008$; Huang and $\mathrm{Lu}, 2010$; Li et al., 2012; Liu et al., 2006) and pesticide residues (Del Fiore et al., 2010; Peng et al., 2011; Shahin and Symons, 2011). Thus, this technology may have the potential to be used as an alternative method for predicting the MCU of materials during drying.

In this study, we used maize kernels as raw materials to process dried products and evaluate the MCU during drying based on hyperspectral reflectance imaging technology. The specific objectives are as follows:

- to extract image traits from preprocessed hyperspectral reflectance images of dried maize kernels using the mean method; and

- to evaluate the capability of partial least squares regression (PLSR) models for predicting the MCU of maize kernels during drying.

\section{MATERIALS AND METHODS}

Fresh maize (Zea mays L.) kernels used in this study, harvested at the commercial state, were purchased from a local market. To achieve a broader distribution of MC and reduce non-uniformity of initial MC, the maize kernels were soaked in water for $12 \mathrm{~h}$. Then they were drained and kept at $4{ }^{\circ} \mathrm{C}$ and $95 \%$ relative humidity in a refrigerator and were used within 3 days. Fresh samples were kept at room temperature $\left(\sim 24{ }^{\circ} \mathrm{C}\right)$ for one hour before the experiment was started.

Maize kernels were dried using a high-precision laboratory dryer developed at the State Key Laboratory of Food Science and Technology, Jiangnan University, China (Wang et al., 2013b). This microwave-assisted pulse-spouted bed vacuum- drying (PSMVD) experimental system essentially included a cylindrical multimode microwave cavity, a circular duct vacuum drying chamber, a pulse-spouted system, a heat supply system, a vacuum system, and a water load system. A detailed description of the dryer system is given by Wang et al. (2013b).

In this study, the experimental parameters were set as follows:

- the pressure was set at 7-10 $\mathrm{kPa}$,

- the power was set to $516 \mathrm{~W}$, and

- the samples were spouted in the preselected time interval of $5 \mathrm{~s}$ and held for $3 \mathrm{~s}$ by allowing nitrogen gas to flow periodically into the drying chamber.

Fresh maize kernels with a mass of $200 \mathrm{~g}$ were used for each batch. To achieve broad sample distribution of MC, six batches at different drying times (from 10 to 60, in steps of $10 \mathrm{~min}$ ) were tested. The experiments were replicated thrice for each drying time. For each batch, two hundred dried maize kernels were selected randomly for hyperspectral imaging and then tested using reference methods for MC. In total, 3600 kernels were used for further analysis.

An in-house developed line-scan hyperspectral reflectance imaging system was used to acquire hyperspectral reflectance images from the maize kernels. The system includes a back-illuminated $1392 \times 1024$-pixel CCD (charge-coupled device) camera (Pixelfly QE IC 285AL, Cooke, USA), a spectrograph (1003A-10140 Hyperspc ${ }^{\mathrm{TM}}$ VNIR C-Series, Headwall Photonics Inc., Fitchburg, USA) with a $25 \mu \mathrm{m}$ slit covering an effective range of $400 \mathrm{~nm}$ to $1000 \mathrm{~nm}$, a zoom lens (10004A-21226 Lens, F/1.4 FL23 mm, Standard Barrel, C-Mount., USA), a single optic line-light powered by a $150-\mathrm{W}$ DC light source (halogen lamp, EKE, $3250 \mathrm{~K}$, Techniquip, USA), and a horizontal motorized stage. Ten maize kernels were placed onto a $20 \times 20 \mathrm{~cm}$ black background board in two rows and perpendicular to the scanning line of the hyperspectral imaging unit. Coupled with the distance between the zoom lens and the sample $(25 \mathrm{~cm})$, a $35 \mathrm{~mm}$ scan length of longitudinal and a $80 \mu \mathrm{m}$ horizontal step size parameters were preset to acquire the whole undistorted image of one group samples.

For each group of samples, 438 scans covering a $35 \mathrm{~mm}$ distance were acquired at an exposure time of $110 \mathrm{~ms}$ for each hyperspectral reflectance image. The hyperspectral imaging system had $0.15 \mathrm{~mm} /$ pixel spatial resolution and a $0.644 \mathrm{~nm}$ spectral interval using a 1392 pixel camera. After 10 spectral binning operations, the resultant hyperspectral reflectance images had a $6.44 \mathrm{~nm}$ spectral interval and 94 wavelengths. Thus, a spatial block of a 1,392 438 $\times 94$ image was created, which was represented by a 2-D image with $\mathrm{x}$-axis and $\mathrm{y}$-axis coordinate information. Another axis was represented by spectral information. Darkness and reflectance images of white Teflon were also acquired for all the five groups of samples and used as reference to obtain relative reflectance images. All the acquired images were completed by Hyperspectral Scanning and Image Rendering Software, Rev a.2.1.3 (Headwall Photonics, Inc., USA).

The MC, expressed in on a percent wet basis (\% w.b.), was measured by the gravimetric method using a convection oven. The samples dried at different times using the PSMVD experimental system were placed into the oven (Binder FED, Berlin, Germany) at $105^{\circ} \mathrm{C}$ until they reached a constant weight (Ning, 1997). The weight was measured using an analytical balance (Hengping FA1104, Shanghai, China; $\pm 0.0001 \mathrm{~g}$ ). The MC was measured and MCU was calculated.

The light source variation effect was corrected by obtaining relative reflectance images using the following equation:

$$
T_{R}=\frac{T_{S}-T_{D}}{T_{F}-T_{D}},
$$


where $T_{R}$ is the relative reflectance, $T_{S}$ and $T_{F}$ are the absolute reflectance of the sample and of the reference (ie Teflon), respectively; and $T_{D}$ is the dark signal for the CCD detector. Thus, all further analyses were conducted on the relative reflectance images.

Segmentation of the maize kernels from the hyperspectral image background is a key step in extracting the image features used to develop the prediction models. Among the image segmentation methods, the global threshold approach is a traditional technique with the advantages of easy calculation and high efficiency.

After the automatic segmentation of the image background at each wavelength, a large amount of spatial and spectral information was obtained from the true image of each maize kernel. Image analysis mainly aims at effective extraction of useful information. Considering the apparent changes in the surface of the maize kernels and the MC of the maize kernels during the drying process, mean reflectance was applied to extract and predict the MCU of the dried grains during the drying process. Similar to the near-infrared spectrum, the mean value of relative reflectance images (mean of the i pixel intensity in the image at each wavelength) provides physical and chemical information on the samples. The mean reflectance is expressed in Eq. (2):

$$
\bar{R}=\frac{1}{M N} \sum_{i=1}^{M} \sum_{i=1}^{N} T_{R}(i, j),
$$

where $\bar{R}$ is mean reflectance, $T_{R}(i, j)$ is the relative reflectance intensity of the pixel $(i, j),(i=1,2 \ldots M, j=1,2 \ldots N)$; and $M, N$ are the number of total horizontal and vertical pixels for a maize kernel, respectively.

PLSR was used to predict the MCU using the mean reflectance feature parameter. In this study, two methods were investigated including indirect prediction and direct prediction uniformity. In the indirect method, MC was adopted as a reference variable to develop the calibration model and the prediction value of MC was used to calculate the MCU, which was investigated if one of the models could be used to predict the MC and MCU simultaneously. Two hundred maize kernels for each drying time $(10,20$, $30,40,50$, and $60 \mathrm{~min}$ ) were randomly divided into two sets: $3 / 4$ of the samples were used for calibration and the remaining $1 / 4$ was used for prediction (independent validation). The calibration sets from each drying time made up the whole calibration set and the prediction sets from each drying time made up the whole prediction set for predicting the MC of the maize kernels, respectively. For evaluating the performance of the indirect model, the MC prediction values of 10,30, and 50 grains in the prediction set for each drying time were selected in one subset to calculate the MCU, respectively.

Unlike in the indirect method, MCU was adopted as a reference variable in the direct method. In order to compare it with the indirect model, the same amount of samples (10,
30 , and 50 grains) was selected in one subset to calculate the MCU as a reference, then the calibration model was developed using the mean reflectance spectra and the MCU reference directly.

For both methods, the PLSR models (ie selection of the appropriate number of latent variables) were determined by a full cross-validation of the calibration samples using leave-one-out cross validation until the root-meansquare error of cross validation (RMSECV) reached the minimum. After the calibration model was developed, this model was used to predict the independent set of samples that had not been used in the calibration. The calibration and prediction procedure described above was repeated 10 times by selecting a random set of samples. The average values of the correlation coefficient (ie $R_{C}, R_{C V}$, and $R_{P}$ ) and root-mean-square error for calibration and prediction (ie RMSEC, RMSECV, and RMSEP) were calculated to evaluate the performance of the models. The PLSR was run in Matlab (2009b) with PLS-Toolbox 5.0 (Eigenvector Research, Inc., Wenatchee, WA, USA).

\section{RESULTS AND DISCUSSION}

Table 1 shows the statistics for the gravimetric method measurement of $\mathrm{MC}$ for fresh and dried maize kernels at different drying times (from 10 to $60 \mathrm{~min}$ ).

Figure 1 shows the contour segmentation result of representative dried maize kernels $(10,30$, and $50 \mathrm{~min})$ at a $718.2 \mathrm{~nm}$ wavelength.

Figure 2 shows the representative colour images of fresh and dried maize kernels with apparent differences in MC. The samples with higher MC appear smooth and rounded with a homogenous glossy surface. As the drying time increases, the MC decreases, thus causing the samples to have textured surfaces. Shrinkage also occurs as the maize samples lose MC.

Figure 3 shows the representative relative reflectance spectra for fresh and processed maize kernels at different drying times $(10,20,30,40,50$, and $60 \mathrm{~min})$. A typical downward peak was observed at $960 \mathrm{~nm}$, corresponding to $\mathrm{MC}$ absorption. Along the increase in the drying time, the absorption peak gradually disappeared because of the MC loss. Over the full wavelength region, the relative reflectance for the fresh maize kernels was generally greater than that for the dried sample. For the wavelength range from 400 to $920 \mathrm{~nm}$, an evident decrease in relative intensity was observed at the initial drying periods between 10 and $30 \mathrm{~min}$. Thereafter, the intensity of reflected light tended to increase as observed between 40 and 60 min of drying.

At the initial periods of drying, microstructural changes occurred on the surface of the maize kernels because of the evaporation of moisture in the immediate surroundings of the grain surface. During this initial period, vapour diffusion is the predominant mechanism, and the rate of evaporation remains constant, thus resulting in textural 
T a b l e 1. Statistics of the MC measurements for fresh and dried maize kernels

\begin{tabular}{cccccc}
\hline $\begin{array}{c}\text { Drying time } \\
(\mathrm{min})\end{array}$ & $\begin{array}{c}\text { Max } \\
(\%)\end{array}$ & $\begin{array}{c}\text { Min } \\
(\%)\end{array}$ & $\begin{array}{c}\text { Mean } \\
(\%)\end{array}$ & $\begin{array}{c}\text { Standard deviation } \\
(\%)\end{array}$ & $\begin{array}{c}\text { RSD } \\
(\%)\end{array}$ \\
\hline 0 (Fresh) & 79.51 & 74.46 & 77.36 & 0.98 & 1.27 \\
10 & 71.69 & 61.69 & 66.63 & 1.91 & 2.87 \\
20 & 62.03 & 48.74 & 55.66 & 2.59 & 4.65 \\
30 & 44.35 & 27.41 & 34.94 & 3.49 & 9.99 \\
40 & 21.49 & 11.19 & 15.67 & 2.53 & 16.14 \\
50 & 12.86 & 7.13 & 9.89 & 1.17 & 11.83 \\
60 & 10.15 & 5.95 & 8.32 & 0.65 & 7.81 \\
\hline
\end{tabular}
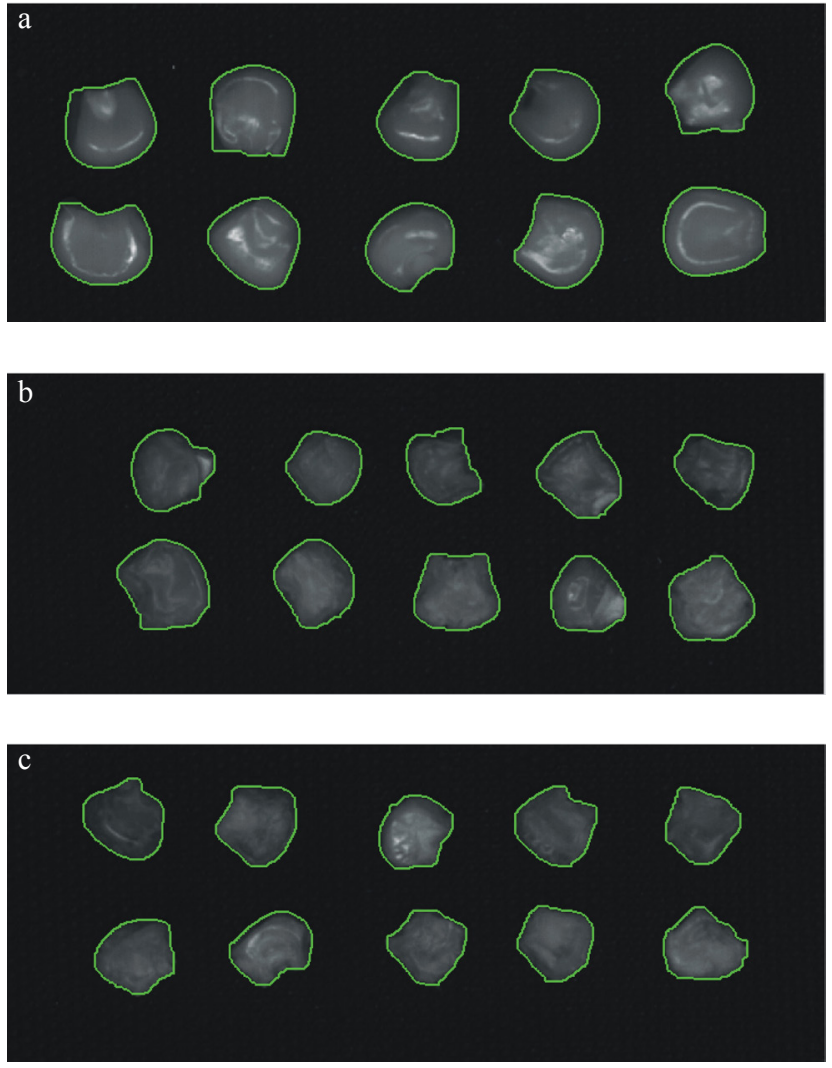

Fig. 1. Contour segmentation results for dried maize kernels: $\mathrm{a}-10, \mathrm{~b}-30, \mathrm{c}-50 \mathrm{~min}$ at a wavelength of $718.2 \mathrm{~nm}$.

changes on the grain surface going from slight to moderate, as observed between 10 and 30 min of drying (Fig. 2b to $2 \mathrm{~d})$. However, as soon as the outer layers of the grain cells on the surface become 'unsaturated' with moisture, the drying rate falls sharply because the diameters of pores and capillaries decrease. This condition results in shrinkage and compactness of the surface microstructure. This compact and brittle structure of the maize kernel probably explains the increase in relative intensity values observed at 40 and 60 min of drying (Fig. 3).
The indirect method, which used the prediction value of MC to calculate the uniformity, was analysed in this study. Mean reflectance spectra were used to develop the MC prediction model coupled with the PLSR algorithm. The MC prediction results are shown in Table 2. The average of 10 calibration and prediction models for $\mathrm{MC}$ yielded good results, with $R_{P}=0.992$, RMSEP $=2.89 \%$. The ratio of prediction deviation (RPD), that is, the ratio of the standard error of performance to the standard deviation of the reference data (Huang and $\mathrm{Lu}, 2010$ ), is an essential parameter in evaluating the performance of prediction models. An RPD between 1.5 and 2 indicates that the model can discriminate low from high values of the response variable. A value between 2 and 2.5 indicates that coarse quantitative predictions are possible, whereas a value between 2.5 and 3 or above corresponds to good and excellent prediction accuracy, respectively (Nicolaï et al., 2007). In this study, the RPD value for MC model was larger than 7.0, so these models can be used to predict the MC and calculate its uniformity further.

For this method, a different number was selected in one subset for computation including 10, 30, 50 maize kernels. Table 3 shows the calculation results of MCU used the prediction values of MC. Although the average correlation coefficient, $R_{P}$, of 10 runs increased with the increase in the kernel number in one subset, it was less than 0.6 , and the RPD was only 0.5 . Figure 4 shows the prediction results for MCU versus the actual measurements for one of the 10 runs for 50 maize kernels in one subset.

Since the indirect method yielded poor prediction results for the MCU of dried maize kernels, the direct method was researched, that is, calibration models were developed for describing the relationship between the mean reflectance spectra and MCU directly. The MCU prediction results for the three different numbers of samples in one subset are shown in Table 4. Compared with the indirect method, the direct method yielded better results for the calibration and prediction models. The average correlation, $R_{P}$, of 10 runs was higher by $33.6-62.8 \%$, whereas the average 

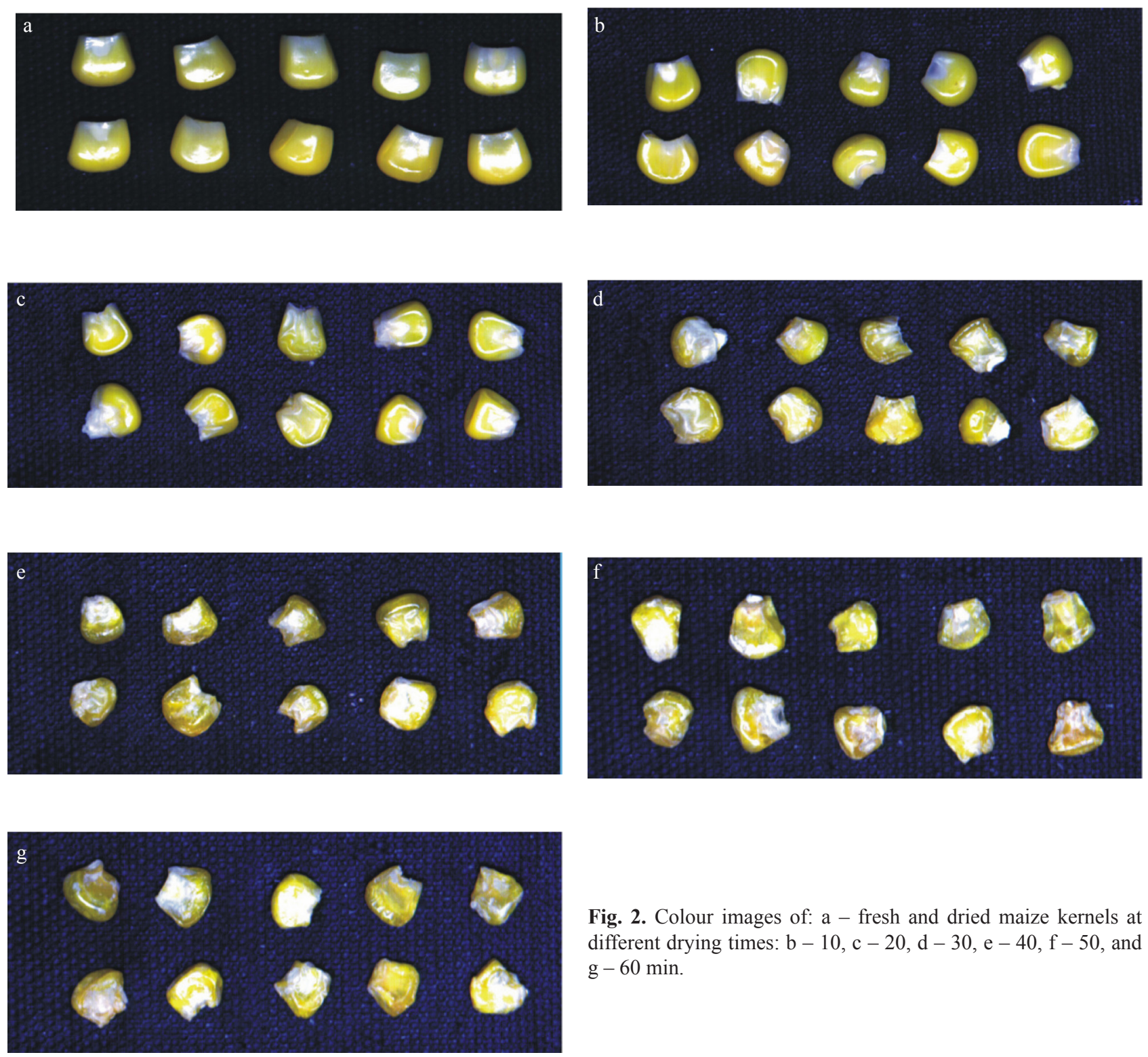

Fig. 2. Colour images of: a - fresh and dried maize kernels at different drying times: $\mathrm{b}-10, \mathrm{c}-20, \mathrm{~d}-30$, e $-40, \mathrm{f}-50$, and $\mathrm{g}-60 \mathrm{~min}$.

RMSEP values were reduced by $54.9-75.1 \%$. The RPD was improved from 0.5 to $1.1,0.5$ to 1.5 , and 0.5 to 1.9 respectively for the different numbers of samples. Figure $5 \mathrm{a}, \mathrm{b}$ show the prediction results for MCU versus the actual measurements for one of the 10 runs for 30 and 50 maize kernels in one subset, respectively.

In this study, a non-destructive detection technique was the first attempt applied to predict the moisture content uniformity of materials in the drying process. Scholars have done valuable work in this field in recent years using the traditional measuring method, such as Wang et al. (2013b), who used the traditional method to calculate the moisture content uniformity. This method can calculate the drying uniformity, but it wastes time and energy. Wang et al. (2014) researched the drying uniformity in radio frequency drying of macadamia nuts; the results show that uniformity needs

very strict conditions. The sample would be damaged and cannot be used again. Compared with the traditional measuring method (Wang et al., 2013b; Wang et al., 2014), hyperspectral imaging technology has rapid, energy-saving, and non-destructive advantages.

This research has demonstrated that the hyperspectral reflectance imaging technique yields good results in predicting the MCU of maize kernels during drying. Compared with the indirect method, direct prediction models yielded superior results for $\mathrm{MCU}$ predictions of dried maize kernels. Although good prediction results for MC were obtained by PLSR models, poor results were obtained for calculating uniformity by the indirect method. The MC was an intermediate variable to calculate the uniformity, and each prediction value had an error corresponding to the actual value. When more prediction values were used to calculate 


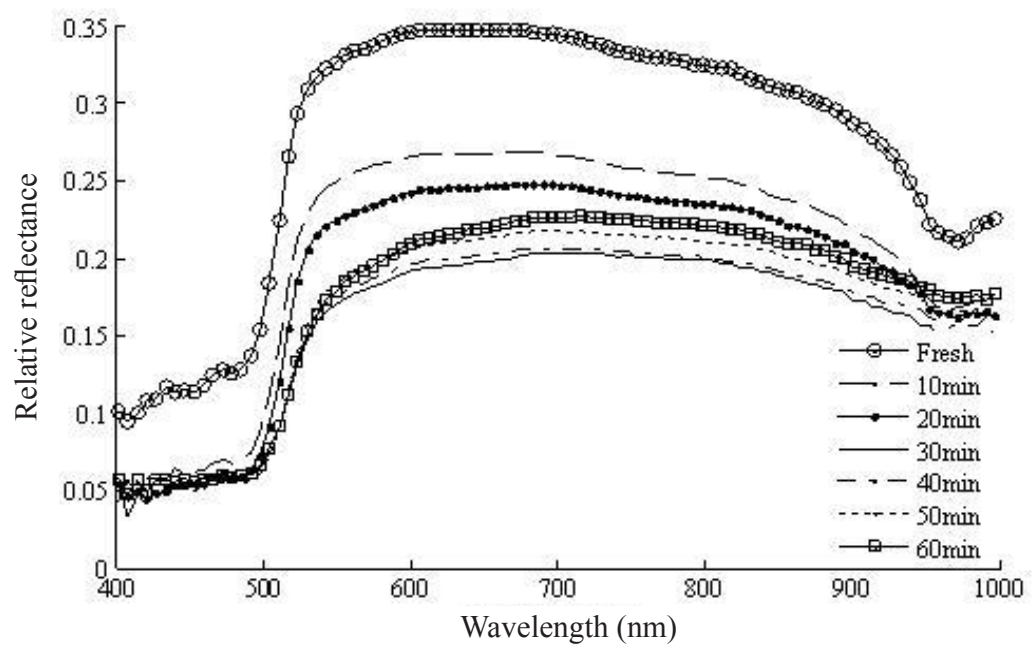

Fig. 3. Relative reflectance for fresh maize kernels at different drying times (10, 20, 30, 40, 50, and 60 min).

T a b l e 2. Average of 10 calibration and prediction results for MC by mean reflectance coupled with PLSR models for dried maize kernels

\begin{tabular}{cccccccc}
\hline LVS & $R_{C}$ & $\begin{array}{c}\text { RMSEC } \\
(\%)\end{array}$ & RCV & $\begin{array}{c}\text { RMSECV } \\
(\%)\end{array}$ & $R_{P}$ & $\begin{array}{c}\text { RMSEP } \\
(\%)\end{array}$ \\
\hline 15 & 0.993 & 2.71 & 0.992 & 3.00 & 0.992 & 2.88 & 7.9 \\
\hline
\end{tabular}

LVs - number of leaves, $R_{C}, R_{C V}$, and $R_{P}$ - correlation coefficient of calibration, cross validation, and prediction, respectively, RMSEC, RMSECV, and RMSEP - root-mean-square error of calibration, cross validation, and prediction, respectively, RPD - ratio of the standard error of performance to the standard deviation of the reference data.

T a b l e 3. Average of 10 calibration and prediction results for MCU calculating by MC prediction values

\begin{tabular}{cccc}
\hline $\begin{array}{c}\text { Number of } \\
\text { maize kernel }\end{array}$ & $R_{P}$ & RMSEP & RPD \\
\hline 10 & 0.324 & 15.17 & 0.5 \\
30 & 0.493 & 11.63 & 0.5 \\
50 & 0.521 & 10.96 & 0.5 \\
\hline
\end{tabular}

Explanations as in Table 2.

the uniformity, the cumulative error resulted in poor uniformity accuracy. Therefore, one model could not predict the MC and MCU of dried maize kernels simultaneously.

Although the models by the direct method yielded better results for $\mathrm{MCU}$, more work has to be done to improve prediction accuracy. Future studies will address wavelength-selection approaches to identify the optimal wavelengths among the 94 wavelengths in order to remove redundant information. Moreover, future works will focus on the evaluation of moisture uniformity within a kernel using hypersectral imaging.

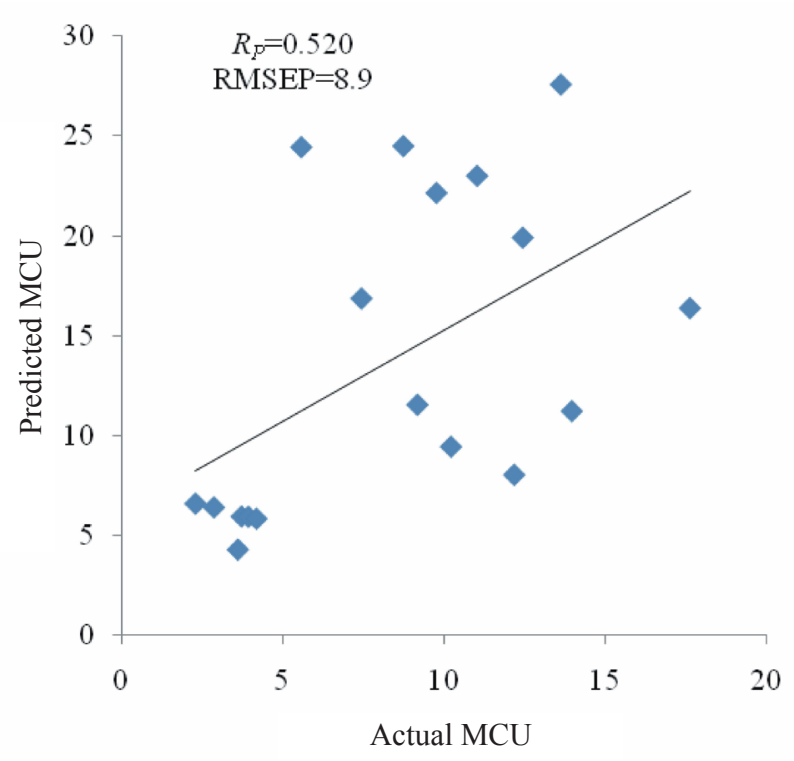

Fig. 4. Prediction of the uniformity of maize kernels using PLSR models by $\mathrm{MC}$ prediction values for 50 maize kernels in one subset. 
T a b l e 4. Average of 10 calibration and prediction results for MCU by mean reflectance coupled with PLSR models for dried maize kernels

\begin{tabular}{ccccccccc}
\hline $\begin{array}{c}\text { Number of } \\
\text { maize } \\
\text { kernel }\end{array}$ & LVs & $R_{C}$ & RMSEC & $R_{C V}$ & RMSECV & $R_{P}$ & RMSEP & RPD \\
\hline 10 & 7 & 0.583 & 5.76 & 0.473 & 6.32 & 0.433 & 6.84 & 1.1 \\
30 & 6 & 0.831 & 2.87 & 0.744 & 3.54 & 0.745 & 3.57 & 1.5 \\
50 & 5 & 0.889 & 2.26 & 0.832 & 2.75 & 0.848 & 2.73 & 1.9 \\
\hline
\end{tabular}

Explanations as in Table 2.

a

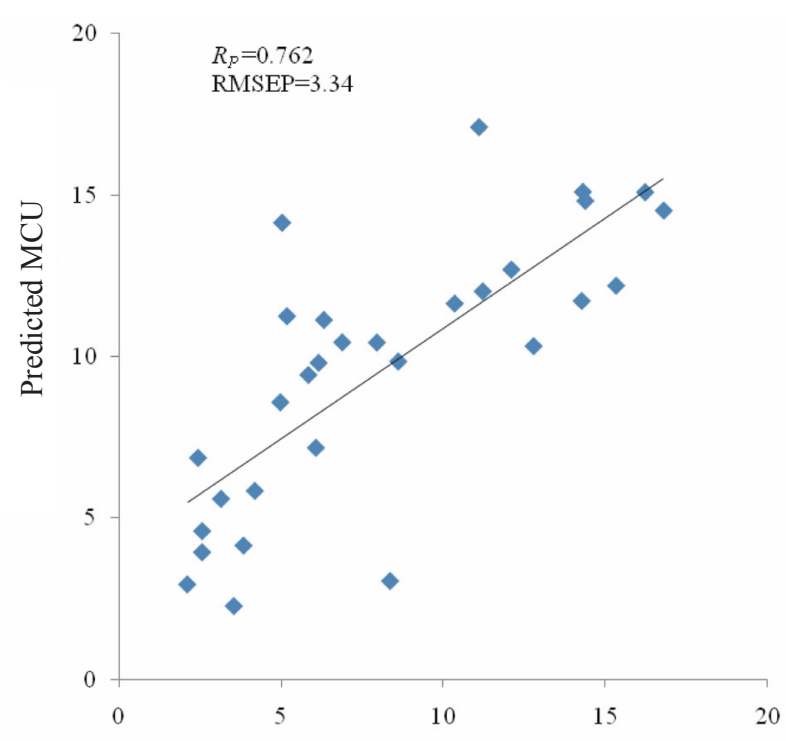

b

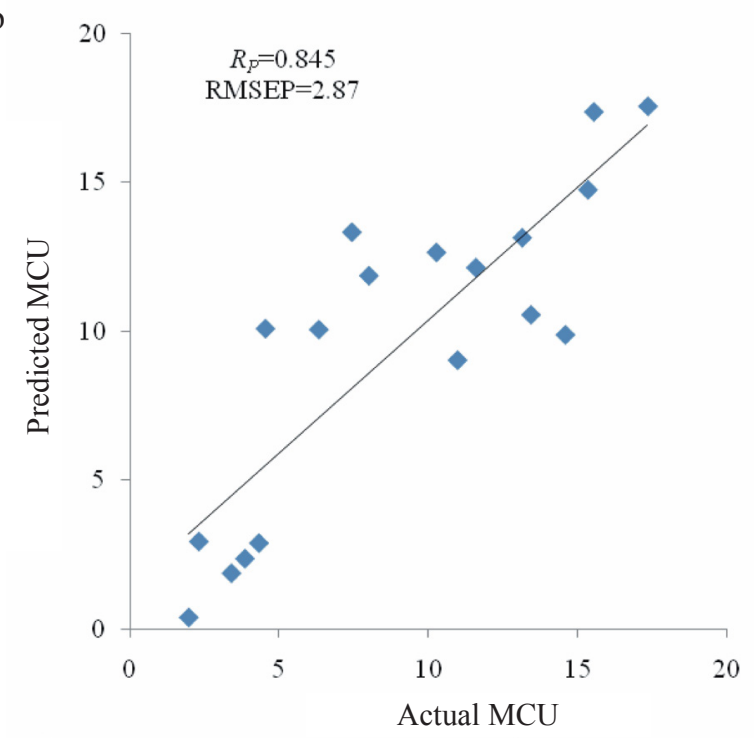

Fig. 5. Prediction of the MCU of maize kernels using PLSR models for: $\mathrm{a}-30$ maize kernels and $\mathrm{b}-50$ maize kernels in one subset.

\section{CONCLUSIONS}

1. The method using the prediction value of moisture content to calculate the uniformity (indirect) was investigated by hyperspectral imaging technology in the wavelength range of $400 \mathrm{~nm}$ to $1000 \mathrm{~nm}$ coupled with partial least squares regression models, which presents really poor results.

2. Compared with the indirect predictions of moisture content uniformity, direct partial least squares regression with mean reflectance yielded better results $\left(R_{P}=0.848\right.$ and root-mean-square error of prediction $=2.73$ ).

3 . The research results indicate that hyperspectral reflectance images over the wavelength range of 400 to $1000 \mathrm{~nm}$ could be used to evaluate the moisture content uniformity of maize kernels during the drying process.

\section{REFERENCES}

Aguilera J.M., 2003. Drying and dried products under the microscope. Food Sci. Technol. Int., 9, 137-143.

Ariana D.P. and Lu R.F., 2008. Quality evaluation of pickling cucumbers using hyperspectral reflectance and transmittance imaging. Part II. Performance of a prototype. Sens. Instrum Food Qual. Saf., 2, 152-160.

Del Fiore A., Reverberi M., Ricelli A., Pinzari F., Serranti S., Fabbri A.A., Bonifazi G., and Fanelli C., 2010. Early detection of toxigenic fungi on maize by hyperspectral imaging analysis. Int. J. Food Microbiol., 144, 64-71.

Faustino J.M.F., Barroca M.J., and Guiné R.P.F., 2007. Study of the drying kinetics of green bell pepper and chemical characterization. Food Bioprod Process., 85, 163-170.

Fernández L., Castillero C., and Aguilera J.M., 2005. An application of image analysis to dehydration of apple discs. J. Food Eng., 67, 185-193.

Hashemi S.J. and Murray Douglas W.J., 2003. Moisture nonuniformity in drying paper: measurement and relation to process parameters. Dry Technol., 21, 329-347.

Huang M. and Lu R.F., 2010. Apple mealiness detection using hyperspectral scattering technique. Postharvest Biol. Tec., 58, 168-175.

Huang M., Wan X.M., Zhang M., and Zhu Q.B., 2013. Detection of insect-damaged vegetable soybean using hyperspectral transmittance image. J. Food Eng., 116, 45-49.

Li J.B., Rao X.Q., and Ying Y.B., 2012. Development of algorithms for detecting citrus canker based on hyperspectral reflectance imaging. J. Sci. Food Agr., 92, 125-134. 
Liu Y., Chen Y.R., Wang C.Y., Chan D.E., and Kim M.S., 2006. Development of hyperspectral imaging technique for the detection of chilling injury in cucumbers; Spectral and image analysis. Appl. Eng. Agric., 22, 101-111.

Lucas A., Andueza D., Rock E., and Martin B., 2008. Prediction of dry matter, fat, $\mathrm{pH}$, vitamins, minerals, carotenoids, total antioxidant capacity, and color in fresh and freeze-dried cheeses by visible-near-infrared reflectance spectroscopy. J. Agr. Food Chem., 56, 6801-6808.

Makky M., Soni P., and Salokhe V., 2014. Automatic nondestructive quality inspection system for oil palm fruits. Int. Agrophys., 28, 319-329

Mendoza F., Dejmek P., and Aguilera J.M., 2006. Calibrated color measurements of agricultural foods using image analysis. Postharvest Biol. Tec., 41, 285-295.

Mireei S.A., Mohtasebi S.S., Massudi R., Rafiee S., and Arabanian A.S., 2010. Feasibility of near infrared spectroscopy for analysis of date fruits. Int. Agrophys., 24, 351-356.

Nicolaï B.M., Beullens K., Bolelyn E., Peirs A., Saeys W., Theron K.I., and Lammertyn J., 2007. Nondestructivemeasurement of fruit and vegetable quality by means of NIR spectroscopy: A review. Postharvest Biol. Tec., 46, 99-118.

Ning Z.X., 1997. Analysis handbook of food component. Chinese Light Industry Publisher, Beijing, China.

Nowak D. and Lewicki P.P., 2005. Quality of infrared dried apple slices. Dry Technol., 23(4), 831-846.

Peng Y.K., Zhang J., Wang W., Li Y.Y., Wu J.H., Huang H., Gao X.D., and Jiang W.K., 2011. Potential prediction of the microbial spoilage of beef using spatially resolved hyperspectral scattering profiles. J. Food Eng., 102, 163-169.
Romano G., Argyropoulos D., Nagle M., Khan M.T., and Müller J., 2012. Combination of digital images and laser light to predict moisture content and color of bell pepper simultaneously during drying. J. Food Eng., 109, 438-448.

Shahin M.A. and Symons S.J., 2011. Detection of fusarium damaged kernels in Canada western red spring wheat using visible/near-infraredhyperspectralimagingandprinciplecomponent analysis. Comput. Electron. Agric., 75, 107-112.

Toyoda K., Tsenkova R.N., and Nakamura M., 2001. Characterization of osmotic dehydration and swelling of apple tissues by bioelectrical impedance spectroscopy. Dry Technol., 19, 1683-1695.

Wang Y.C., Zhang M., Mujumdar A.S., and Mothibe K.J, 2013a. Microwave-assisted pulse-spouted bed freeze-drying of stem lettuce slices-effect on product quality. Food Bioprocess Tech., 6, 3530-3543.

Wang Y.C., Zhang M., Mujumdar A.S., Mothibe K.J., and Roknul Azam S. M., 2013b. Study of drying uniformity in pulsed spouted microwave-vacuum drying of stem lettuce slices with regard to product quality. Dry Technol., 31, 91-101.

Wang Y.Y., Zhang L., Gao M.X., Tang J.M., and Wang S.J., 2014. Pilot-scale radio frequency drying of macadamia nuts: heating and drying uniformity. Dry Technol., 32, 1052-1059.

Wu D., He Y., Nie P.C., Cao F., and Bao Y.D., 2010. Hybrid variable selection in visible and near-infrared spectral analysis for non-invasive quality determination of grape juice. Anal. Chim. Acta., 659, 229-237. 